تعيين عمق بهينه آب آبيارى جغندرقند در شرايط وابستخى قيمت محصول به مقدار آب آبيارى

\author{
على شعبانى

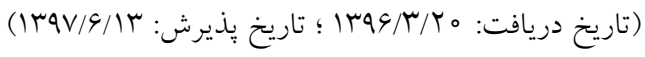

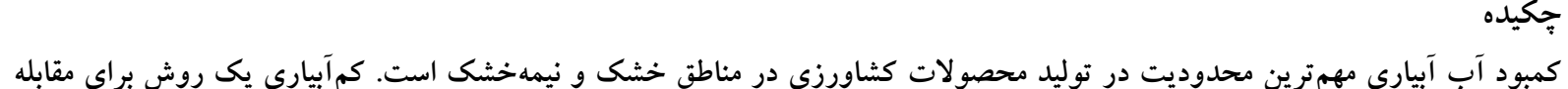

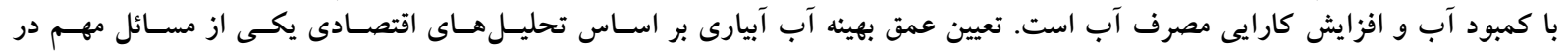

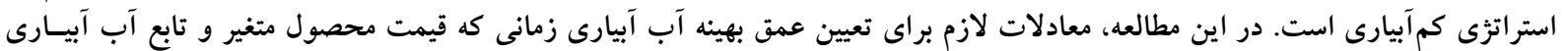

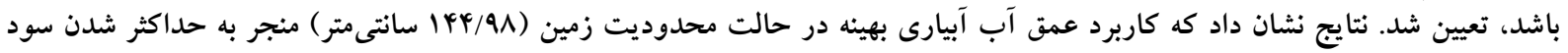

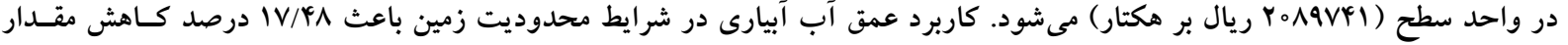

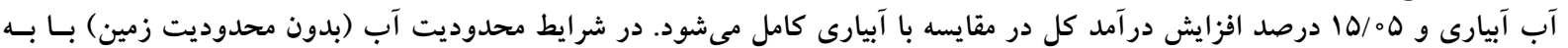

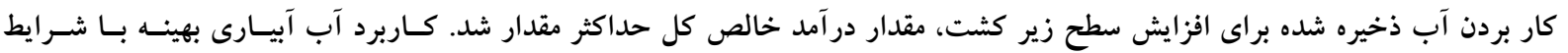

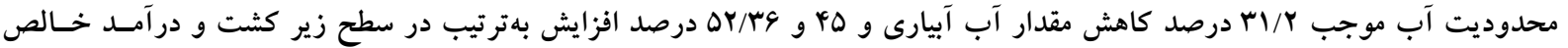

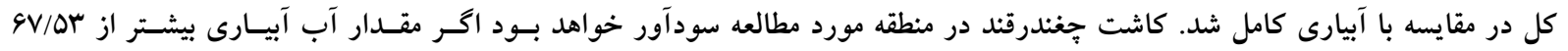




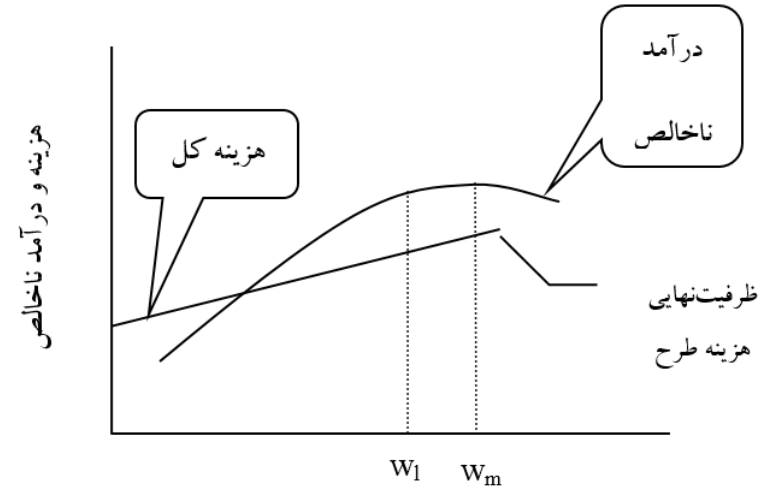

آب به كار رفته

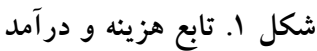

انجام شده در اين زمينه بر تحليل هـاى رياضسى - اقتصـادى كـه

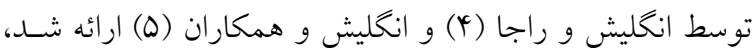

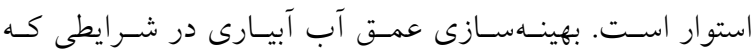

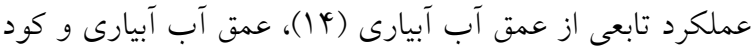
نيتروزنه (IY)، مقدار بارندگى + عمق آب آبيارى (Y (I) و مقدار بارندگى + عمق آب آبيارى و كود نيتروزنسه (rا) باشــ انجـام شده است. در تمام تحليل هاى فوق، قيمت محصول ثابت بـوده

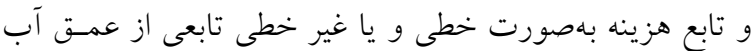

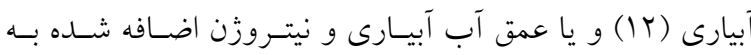

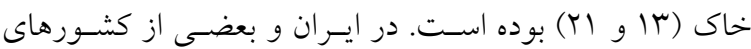

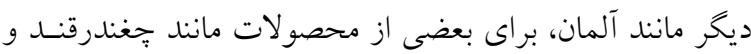

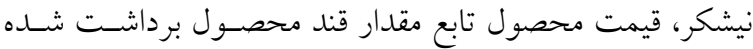

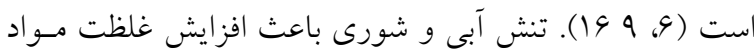

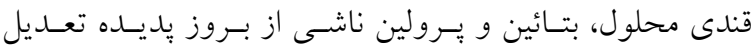

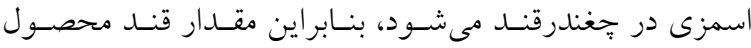

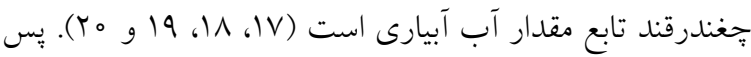

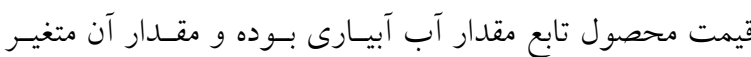

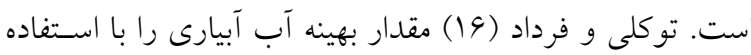

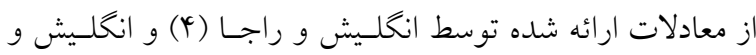

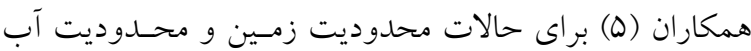

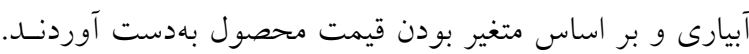

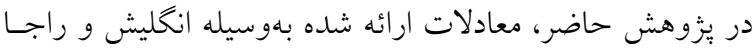

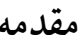

يكى از راهكارهاى ارائه شده براى افزايش كارايى مصـرف آب

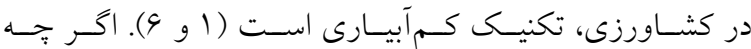
يُزوهشهاى بسيار زيادى در اين زمينه وجود دارد ولى بهعنوان نمونه براى گياه جغنندرقند مى توان به يافتسهاى جهـوزى و زارع

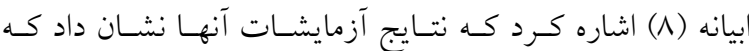

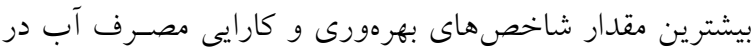
تيمار آبيارى هم درصد كمآبيارى در روش آبيارى بخشى ريشسه مشاهده شد. تار كالسون و كينخ (10) مشاهده كردند كه انجـام

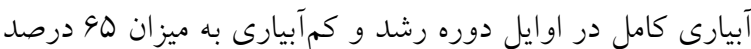

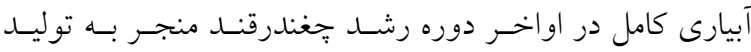

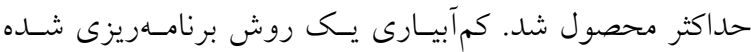

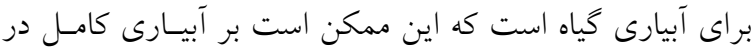

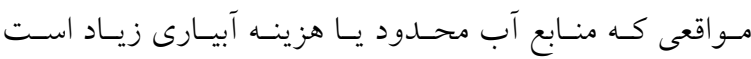
ارجحيت داشته باشد (ه). شكل (1) تغييـرات درآمــ و هزينـه

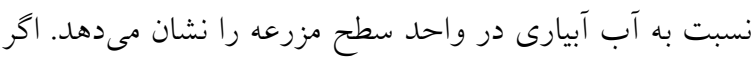

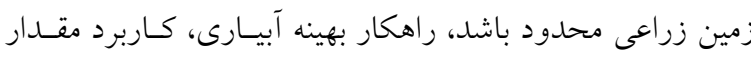

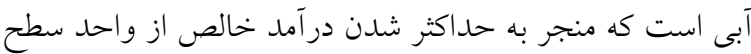

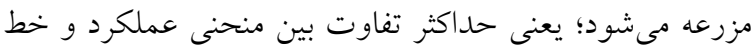

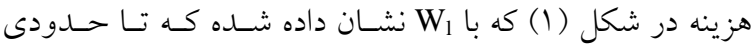

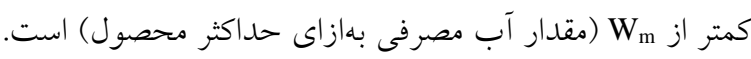
از ديد اقتصادى، اين نقطه محلى است كه شيب تابع هزينه برابر مهرك

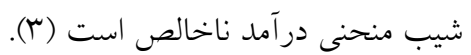

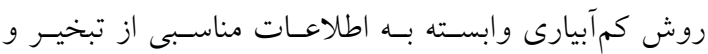

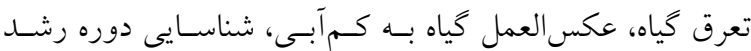
بحرانى كياه و اثرات اقتصادى كاهش محصـول ناشسى از اتخــاذ

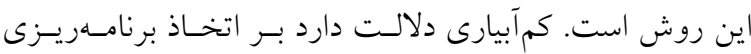

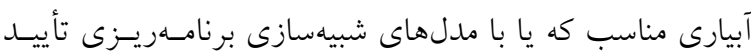

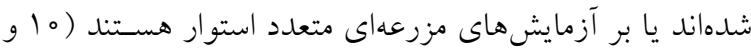

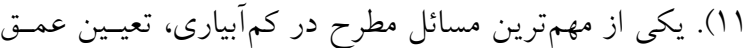

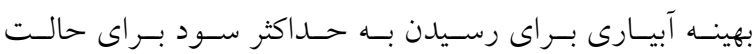
محدوديت زمين و محدوديت آب آبيارى است. عمده مطالعات 
در شرايطى كه زمين عامل محدود كننده باشد، سطح زير كشت

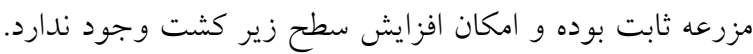

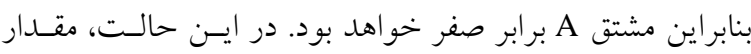
عمق بهينه آب آبيارى از رابطه زير محاسبه مى بشود: $\frac{\partial \mathrm{i}_{1}(\mathrm{w})}{\partial \mathrm{w}}=$ 。

با قرار دادن معادلـه (ז) در معادلـه (Q) معـادلات زيـر حاصـل

$\frac{\partial\left[\mathrm{p}_{\mathrm{c}} \mathrm{y}(\mathrm{w})-\mathrm{c}(\mathrm{w})\right]}{\partial \mathrm{w}}=。$

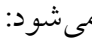

$\mathrm{p}_{\mathrm{c}} \frac{\partial \mathrm{y}(\mathrm{w})}{\partial \mathrm{w}}-\frac{\partial \mathrm{c}(\mathrm{w})}{\partial \mathrm{w}}=。$

در شرايط محدوديت آب آبيارى، بهدليل اينكه سطح زير كشت

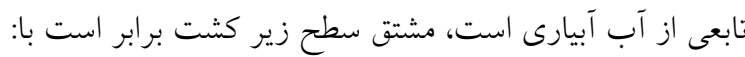
$\frac{\partial \mathrm{A}}{\partial \mathrm{w}}=-\frac{\mathrm{W}_{\mathrm{T}}}{\mathrm{w}^{r}}$

در اين شرايط، با قرار دادن معادلات (Y) و (N) در معادله (Y)،

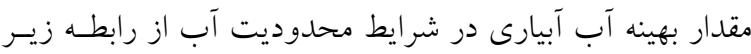

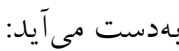

$$
\begin{aligned}
\frac{\mathrm{W}_{\mathrm{T}}}{\mathrm{w}} & {\left[\mathrm{p}_{\mathrm{c}} \frac{\partial \mathrm{y}(\mathrm{w})}{\partial \mathrm{w}}-\frac{\partial \mathrm{c}(\mathrm{w})}{\partial \mathrm{w}}\right]+} \\
& {\left[\mathrm{p}_{\mathrm{c}} \mathrm{y}(\mathrm{w})-\mathrm{c}(\mathrm{w})\right]\left[-\frac{\mathrm{W}_{\mathrm{T}}}{\mathrm{w}^{r}}\right]=。 }
\end{aligned}
$$

با ساده كردن معادله (9): (9) - (9)

$\mathrm{w}\left[\mathrm{p}_{\mathrm{c}} \frac{\partial \mathrm{y}(\mathrm{w})}{\partial \mathrm{w}}-\frac{\partial \mathrm{c}(\mathrm{w})}{\partial \mathrm{w}}\right]=\left[\mathrm{p}_{\mathrm{c}} \mathrm{y}(\mathrm{w})-\mathrm{c}(\mathrm{w})\right]$

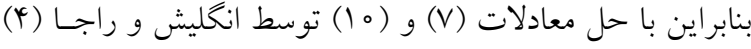

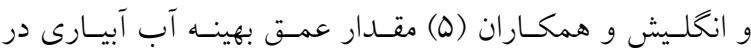

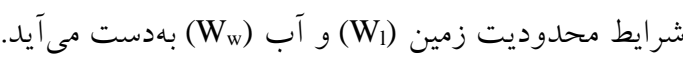
اما همانطور كه تو كلى و فرداد (19) نشان دادهاند، براى بعضى

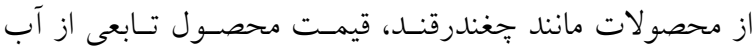

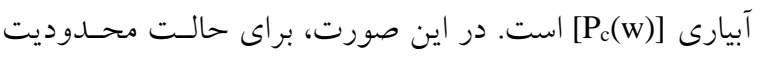
زمين، معادله (G) به صورت زير اصلاح مى بشود: $\mathrm{p}_{\mathrm{c}}(\mathrm{w}) \frac{\partial \mathrm{y}(\mathrm{w})}{\partial \mathrm{w}}+\frac{\partial \mathrm{p}_{\mathrm{c}}(\mathrm{w})}{\partial \mathrm{w}} \mathrm{y}(\mathrm{w})-\frac{\partial \mathrm{c}(\mathrm{w})}{\partial \mathrm{w}}=。$

و براى حالت محدوديت آب، با قرار دادن معسادلات (T) و (^)

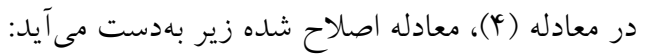

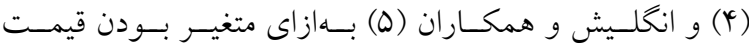

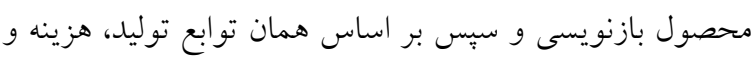

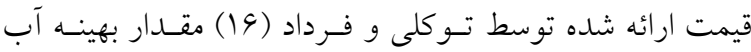
آبيارى در شرايط محدوديت زمين و آب آبيارى مجدداً محاسبه و با نتايج تو كلى و فرداد (19) مقايسه شده است مين.

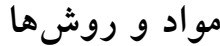

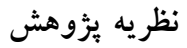

بر اساس تحليل رياضى-اقتصادى كه توسط انخلسيش و راجـا

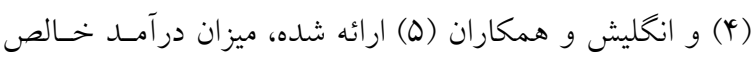
مزرعه از رابطه زير بهدست مى آيد: $\mathrm{I}_{\mathrm{f}}(\mathrm{w})=\mathrm{A} \cdot \mathrm{i}_{\mathrm{l}}(\mathrm{w})$

$\mathrm{i}_{\mathrm{l}}(\mathrm{w})=\left[\mathrm{p}_{\mathrm{c}} \mathrm{y}(\mathrm{w})-\mathrm{c}(\mathrm{w})\right]$

كه در آن، Iآن درآمد خالص مزرعه از كل اراضى آبيارى شده،

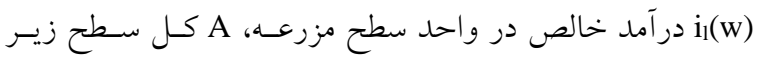
كشت مزرعه (هكتار)، Pيمت و واحد وزن محصول (ريـال بـر

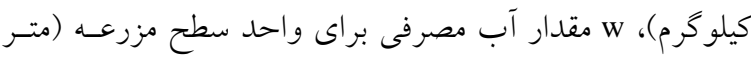

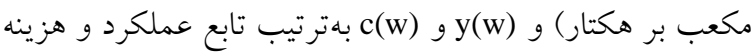

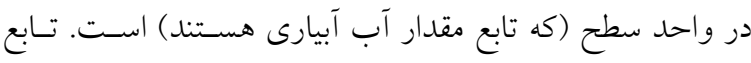

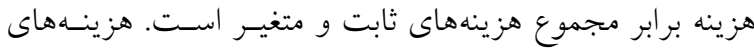

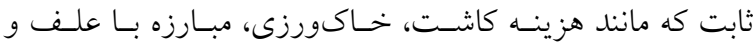

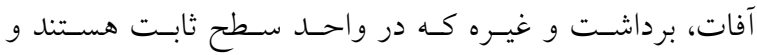

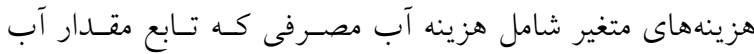
آبيارى است.

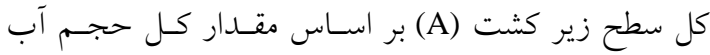

$$
\text { تأمين شده (WT) برابر است با: }
$$

$A=\frac{W_{T}}{W}$

براى تعيين عمق بهينه آب مصرفى، مسىبايست سـود حسداكثر

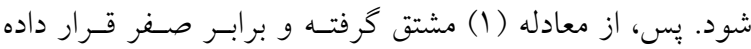

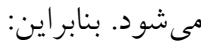

$\frac{\partial \mathrm{I}_{\mathrm{f}}(\mathrm{w})}{\partial \mathrm{w}}=\mathrm{A} \cdot \frac{\partial \mathrm{i}_{1}(\mathrm{w})}{\partial \mathrm{w}}+\mathrm{i}_{\mathrm{l}}(\mathrm{w}) \frac{\partial \mathrm{A}}{\partial \mathrm{w}}=。$ 
محدوديت زمين مطرح است. مقدار Wel از رابطه زيــر محاسبه

$\mathrm{p}_{\mathrm{c}}\left(\mathrm{w}_{\mathrm{el}}\right) \mathrm{y}\left(\mathrm{w}_{\mathrm{el}}\right)-\mathrm{c}\left(\mathrm{w}_{\mathrm{el}}\right)=$

$$
\mathrm{p}_{\mathrm{c}}\left(\mathrm{w}_{\mathrm{m}}\right) \mathrm{y}\left(\mathrm{w}_{\mathrm{m}}\right)-\mathrm{c}\left(\mathrm{w}_{\mathrm{m}}\right)
$$

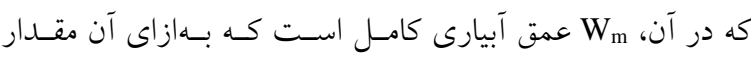

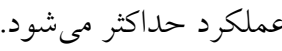

$\frac{\partial \mathrm{y}(\mathrm{w})}{\partial \mathrm{w}}=$ 。

و همجنين عمق معادل آبيارى كامل در حالـت محـدوديت آب عمق آب آبيارى است كـه در آن سـود خـالص بـهازاى (Wew واحد آب مصرفى برابر سود خالص در واحس آبـ آب آبيارى كامـل است كه از رابطه زير محاسبه مىشود: $\frac{p_{\mathrm{c}}\left(\mathrm{w}_{\mathrm{ew}}\right) \mathrm{y}\left(\mathrm{w}_{\mathrm{ew}}\right)-\mathrm{c}\left(\mathrm{w}_{\mathrm{ew}}\right)}{\mathrm{w}_{\mathrm{ew}}}=$

$\frac{\mathrm{p}_{\mathrm{c}}\left(\mathrm{w}_{\mathrm{m}}\right) \mathrm{y}\left(\mathrm{w}_{\mathrm{m}}\right)-\mathrm{c}\left(\mathrm{w}_{\mathrm{m}}\right)}{\mathrm{w}_{\mathrm{m}}}$

در اقتصاد، شرايطى وجود دارد كه از آن تحت عنوان حالت سر بهسرى نام برده مىشود. در اين حالت، مقدار سود برابر صفر و

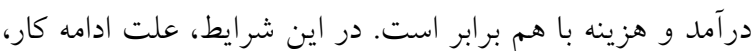

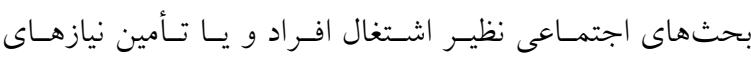

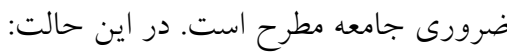
$p_{c}\left(w_{k}\right) y\left(w_{k}\right)=c\left(w_{k}\right)$

تعيين عمقهاى بهينه، عمق آب آبيارى كامل و عمق سـر بسه

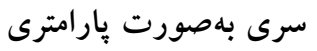
در صورتى كه تابع توليد و هزينه جنغندرقند بهصورت يكى تـابع درجه ب و تابع قيمت بهصورت يكى تابع خطى به فرم معسادلات

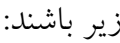

$\mathrm{y}(\mathrm{w})=\mathrm{a}_{1}+\mathrm{b}_{1} \mathrm{w}+\mathrm{c}_{1} \mathrm{w}^{\mathrm{r}}$

$c(w)=a_{Y}+b_{r} w+c_{\Upsilon} w^{r}$

$\mathrm{p}_{\mathrm{c}}(\mathrm{w})=\left(\mathrm{a}_{\mu}+\mathrm{b}_{\mathrm{r}} \mathrm{w}\right) \mathrm{p}_{\mathrm{C} \mid \mathrm{s}}$

كه در آنها Pيمت 列 عمق آبيارى كامل از مساوى صفر قرار دادن مشتق معادلـه (11)

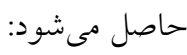

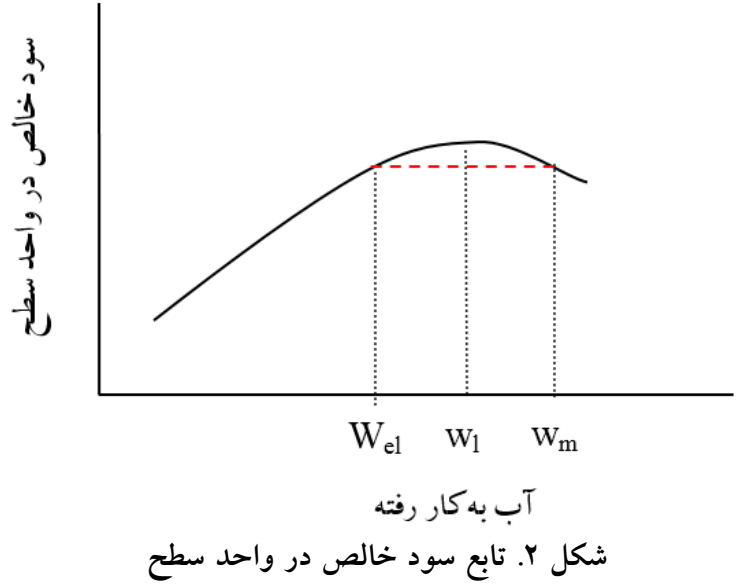

$$
\begin{array}{r}
\frac{\mathrm{W}_{\mathrm{T}}}{\mathrm{w}}\left[\mathrm{p}_{\mathrm{c}}(\mathrm{w}) \frac{\partial \mathrm{y}(\mathrm{w})}{\partial \mathrm{w}}+\frac{\partial \mathrm{p}_{\mathrm{c}}(\mathrm{w})}{\partial \mathrm{w}} \mathrm{y}(\mathrm{w})-\frac{\partial \mathrm{c}(\mathrm{w})}{\partial \mathrm{w}}\right]+ \\
{\left[\mathrm{p}_{\mathrm{c}}(\mathrm{w}) \mathrm{y}(\mathrm{w})-\mathrm{c}(\mathrm{w})\right]\left[-\frac{\mathrm{w}_{\mathrm{T}}}{\mathrm{w}^{\mathrm{r}}}\right]=0}
\end{array}
$$

با ساده كردن معادله (r|)، مقدار بهينـه آب آبيـارى در شـرايط محدوديت آب و ثابت نبودن قيمت محصول با حل معادله زيـر

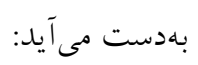

$\begin{array}{r}w\left[p_{c}(w) \frac{\partial y(w)}{\partial w}+\frac{\partial p_{c}(w)}{\partial w} y(w)-\frac{\partial c(w)}{\partial w}\right] \\ {\left[p_{c}(w) y(w)-c(w)\right]}\end{array}=$

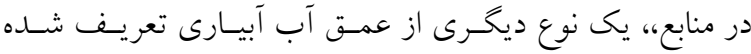
تحت عنوان عمق معادل آبيارى كامل در حالت محدوديت زمين كه در اين عمق سود خالص در واحد سطح در اين عمق (Wel)

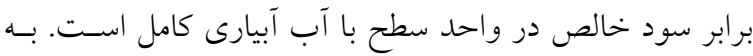

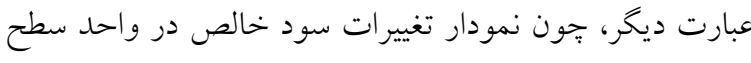

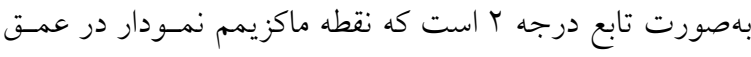

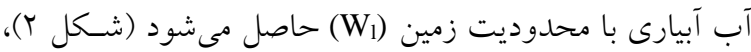

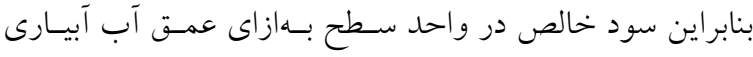
كامل (Wm)، مقدار كمتـرى اسـت كـه در سـمت راسـت نقطـه ماكزيمم نمـودار حاصـل مىشـود. بنـابراين عمسق آب آبيـارى ديخرى در سمت جِّ نمودار هم وجود دارد كه مقدار آن كمتر از عمق آب آبيارى در شرايط محدوديت زمسين اسـت ولسى بــا همان مقدار سود در واحد سطح. تأكيد مىشود كه تمـامى ايسن

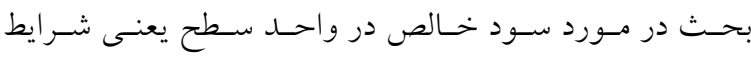




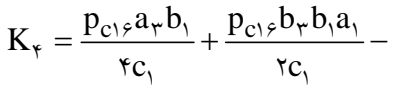

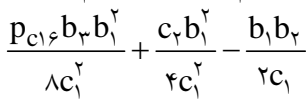

$$
\begin{aligned}
& \text { حل معادله زير تعيين مىشود: } \\
& \mathrm{S}_{1} \mathrm{~W}_{\mathrm{eW}}^{r}+\mathrm{S}_{\mathrm{r}} \mathrm{W}_{\mathrm{eW}}^{r}+\mathrm{S}_{r} \mathrm{~W}_{\mathrm{eW}}+\mathrm{S}_{\mathrm{r}}=。 \\
& \frac{\mathrm{p}_{\mathrm{C}_{\mid \varphi}} \mathrm{b}_{r} \mathrm{~b}_{1}^{r}}{{ }^{r} \mathrm{c}_{1}}-\frac{r \mathrm{a}_{r} \mathrm{c}_{1}}{\mathrm{~b}_{1}}-\frac{\mathrm{b}_{1} \mathrm{c}_{r}}{r \mathrm{c}_{1}}
\end{aligned}
$$

دادههاى مورد استفاده

در اين يزوهش، از توابع توليـد، هزينـهـ و قيمـت ارائسه شــه توسط توكلى و فرداد (19) و اكبرى (Y) براى كياه جغندرقند استفاده شد. يزٔوهش اول در منطقـه كـرج و يـزَوهش دوم در منطقه اصفهان انجـام شـد. بـراى اطلاعـات بيشـتر در مـورد تيمارهاى آزمايشى، ويز كى هاى آبوهوايى و خاك منطقه، به توكلى و فرداد (1)) و اكبرى (Y) مراجعه شود. توابع توليـد، هزينه و قيمت ارائه شده توسط توكلى و فرداد (19) به شرح زير هستند:

$y(W)=-r Y \Delta Q 9 / Y r+9 \Delta r / \Delta D W-r / V I W^{r}$

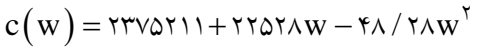

$\mathrm{p}_{\mathrm{C}}(\mathrm{w})=(1 / \mathrm{rr}-0 / 00199 \mathrm{~W}) \mid \mathrm{r} 。$

و توابع توليد، هزينه و قيمت حاصل از مطالعسه اكبـرى (T) بـه شرح زير هستند:

$y(W)=-r V 100 / \Lambda+11 r r / 10 D-r / 9994 r / W^{r}$

$C(W)=r \circ 949 r r+r r 990 / 9 W-9 r / 49 r W^{r}$

$p_{c}(w)=(1 / \wedge \wedge \mu q-\circ / \circ \circ \vee \wedge \wedge w) \mid r \circ$
$\mathrm{W}_{\mathrm{m}}=\frac{-\mathrm{b}_{1}}{\mathrm{rc}_{1}}$

با قرار دادن معادلات (IN) تا (Y०) در معادلـه (IV)، عمـق آب در حالت سربهسرى از حل معادله زير بهدست مى آيد:

$\left(p_{C_{\mid \zeta}} a_{r} a_{1}-a_{r}\right)+\left(p_{C \mid \gamma} a_{r} b_{1}+p_{C \mid \varphi} b_{r} a_{1}-b_{r}\right)$

$\mathrm{w}+\left(\mathrm{p}_{\mathrm{C} \mid \varphi} \mathrm{b}_{r} \mathrm{~b}_{1}+\mathrm{p}_{\mathrm{C} \varphi} \mathrm{a}_{r} \mathrm{c}_{1}-\mathrm{c}_{Y}\right) \mathrm{w}^{r}+$

$\left(\mathrm{P}_{\mathrm{C} \mid \varphi} \mathrm{b}_{r} \mathrm{C}_{1}\right) \mathrm{w}^{r}=0$

در شرايط محدوديت زمين، با قرار دادن معادلات (1/) تا (Yo) و مشتق آنها در معادله (11) معادله زير حاصل مىشود: $\left(\mathrm{p}_{\mathrm{C} \mid \varphi} \mathrm{b}_{r} \mathrm{a}_{Y}+\mathrm{p}_{\mathrm{C} \mid \varphi} \mathrm{a}_{r} \mathrm{~b}_{\uparrow}-\mathrm{b}_{r}\right)+$

$\left(r \mathrm{p}_{\mathrm{C} \mid \varphi} \mathrm{b}_{r} \mathrm{~b}_{1}+r \mathrm{p}_{\mathrm{C} \mid \varphi} \mathrm{a}_{r} \mathrm{c}_{1}-r \mathrm{C}_{r}\right) \mathrm{w}+$

$\left(r \mathrm{p}_{\mathrm{C} \mid \varphi} \mathrm{b}_{r} \mathrm{c}_{1}\right) \mathrm{w}^{r}=$ 。

با حل معادله درجه ب فوق، مقــار عمـق بهينـه آب آبيـارى در شرايط محدوديت زمين (Wl) بهدست مى آيد. با حل اين معادله، دو عمق آب آبيارى بهدست مى آيد. جـواب صسحيح عمـق آب آبيارى است كه مقدار آن كمتر از مقدار عمق آب آبيارى كامـل و بيشتر از عمق آب آبيـارى حالـت سـربسهسـرى (Wh $)$

در شرايط محدوديت آب (WWW)، با قرار دادن معادلات (1N)

تا (Yo) و مشتق آنها در معادله (r) معادله زير حاصل مىشود: $\left(\mathrm{a}_{Y}-\mathrm{p}_{\mathrm{C} \mid \zeta} \mathrm{a}_{r} \mathrm{a}_{1}\right)+\circ / \circ \mathrm{W}+$

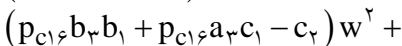

$\left(r \mathrm{p}_{\mathrm{C} \mid \varsigma} \mathrm{b}_{r} \mathrm{c}_{1}\right) \mathrm{w}^{r}=$ 。

معادله درجه ب فوق داراى سـه ريشـه اسـت كـه مانـــد حالـت محدوديت زمين، جواب صحيح عددى است كه مقدار آن كمتر از مقدار عمق آب آبيـارى كامـل و بيشـتر از عمـق آب آبيـارى حالت سربهسرى باشد.

عمق معادل آبيارى كامل در حالت محدوديت زمسين (Wel)

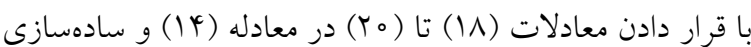

$$
\text { و حل معادله زير تعيين مىشود: }
$$

$\mathrm{K}_{1} \mathrm{~W}_{\mathrm{el}}^{r}+\mathrm{K}_{r} \mathrm{~W}_{\mathrm{el}}^{\Upsilon}+\mathrm{K}_{r} \mathrm{~W}_{\mathrm{el}}+\mathrm{K}_{r}=。$

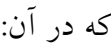

$\mathrm{K}_{1}=\mathrm{p}_{\mathrm{C} \mid \varphi} \mathrm{b}_{r} \mathrm{c}_{1}$

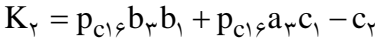

$\mathrm{K}_{r}=\mathrm{p}_{\mathrm{C} \mid \varphi} \mathrm{b}_{r} \mathrm{a}_{1}+\mathrm{p}_{\mathrm{C} \mid \xi} \mathrm{a}_{r} \mathrm{~b}_{1}-\mathrm{b}_{r}$ 


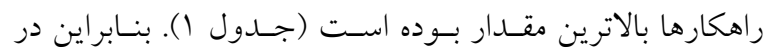

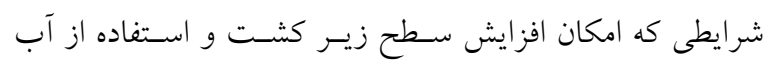

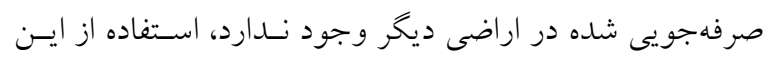
راهكار اقتصادىترين انتخاب است.

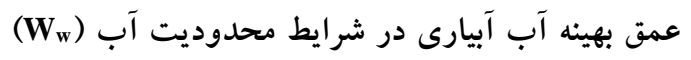

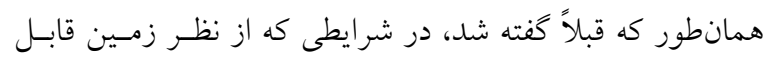

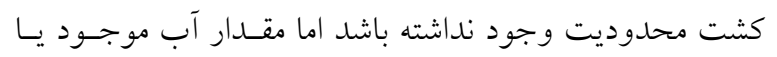

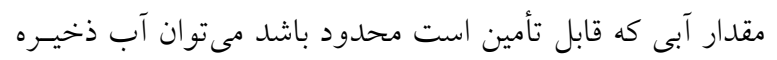

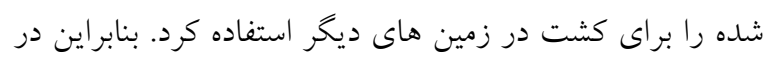

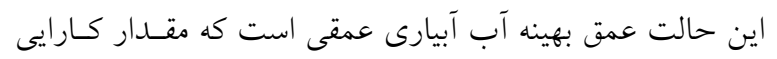

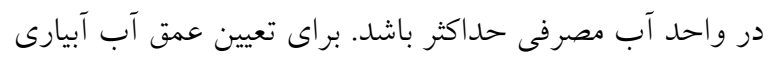

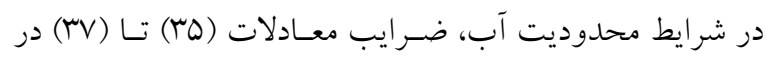

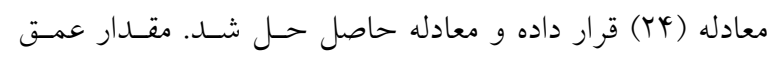

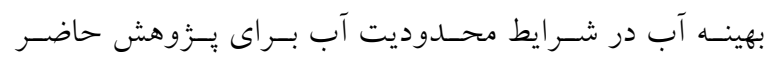
Ko/AY

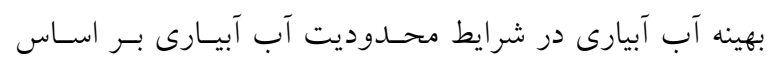

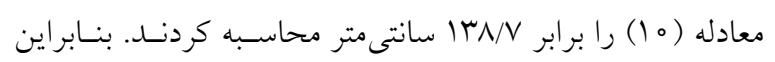
عمق بهينه آب آبيارى محاسبه شده بر اساس معادلا مادلات ارائه شده

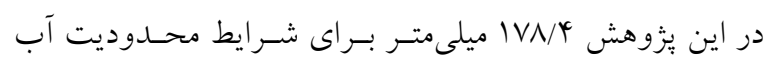

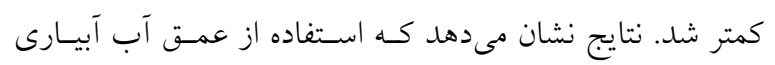

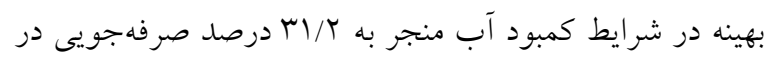

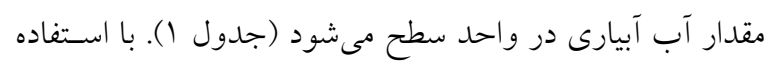

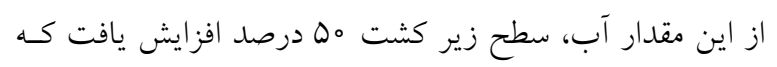

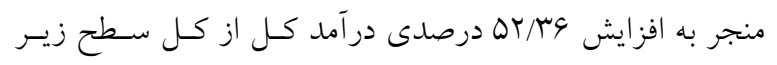

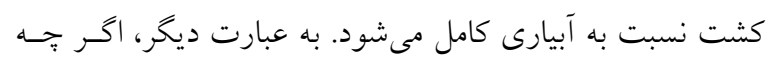

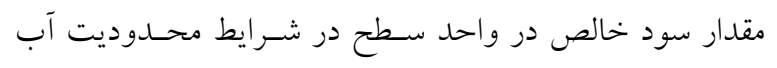

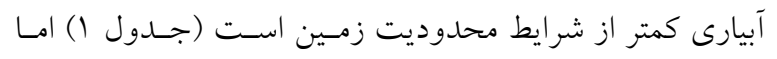

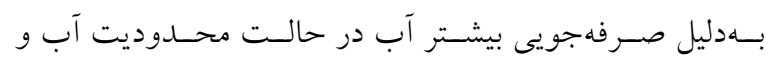

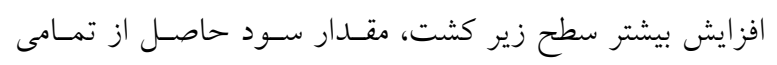

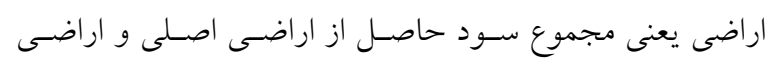

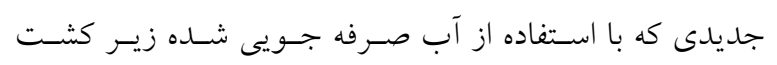

\section{نتايج و بحث} عمق آب آبيارى در شرايط آبيارى كامل (Wm

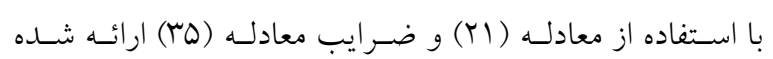

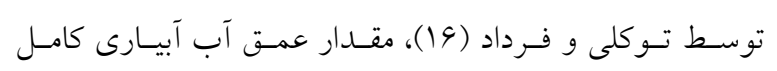

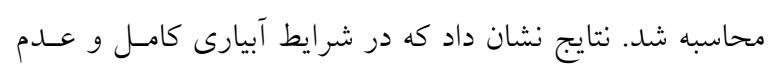

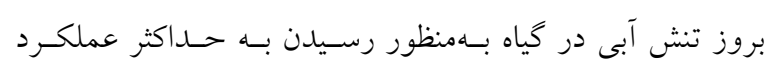

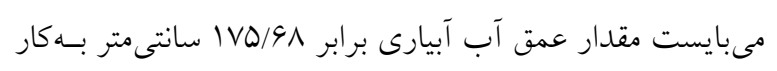

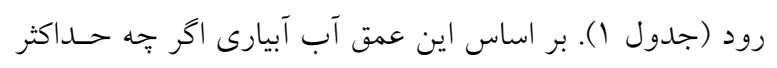

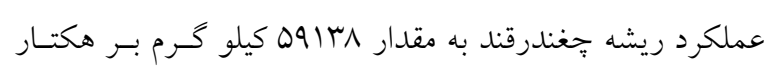

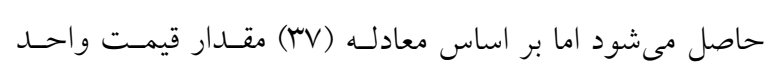

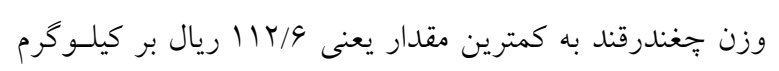
كاهش مى يابد كه بهدليل كاهش مقدار درصد قند ريشه ند ناشى از آبيارى زياد است.

عمق بهينه آب آبيارى در شرايط محدوديت زمين (WI)

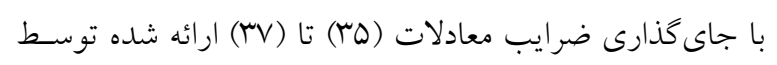

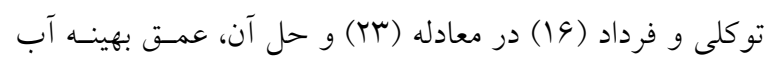

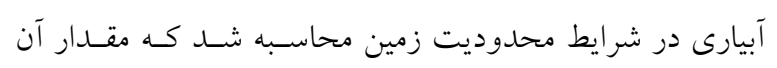

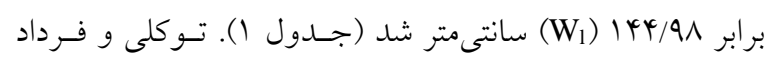

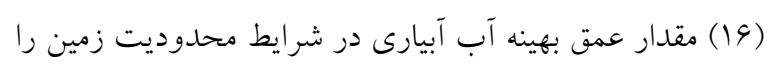

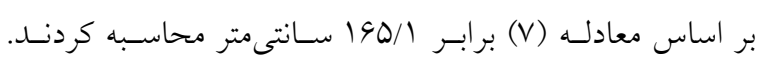

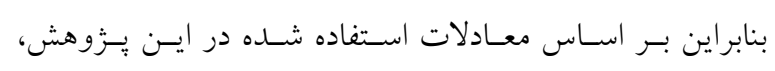

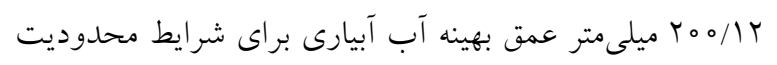

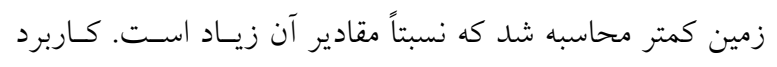

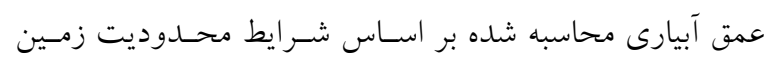

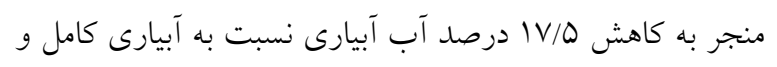

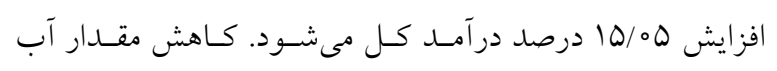

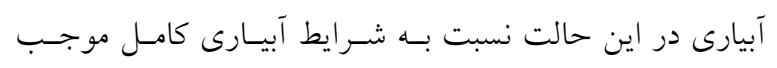

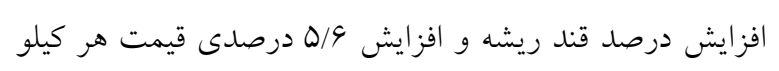

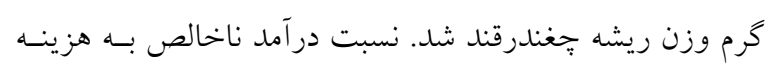

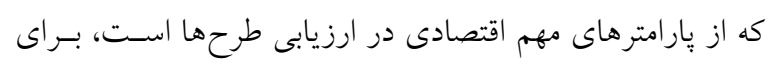

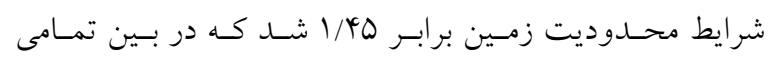




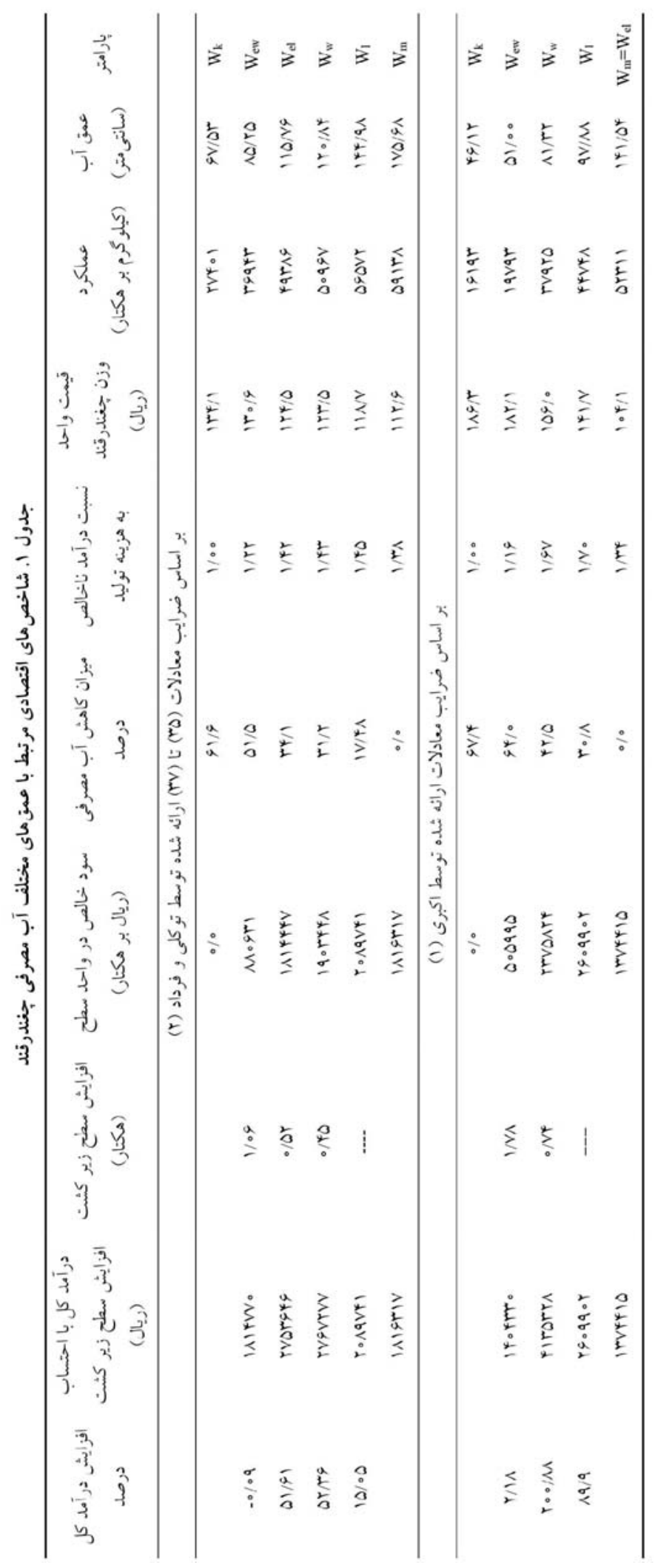


مقــدار عمـق آب آبيـارى معـادل آبيـارى كامـل در شــايط

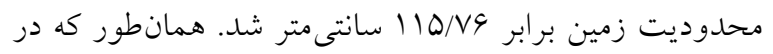

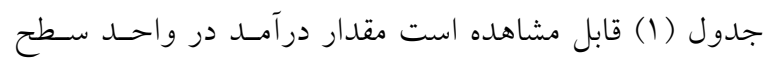

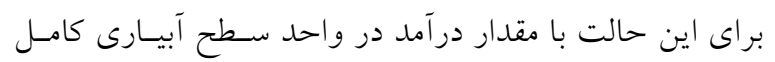

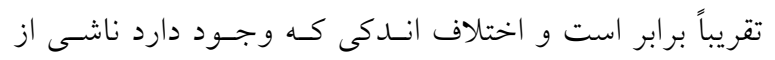

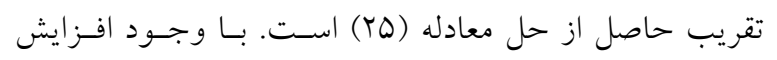

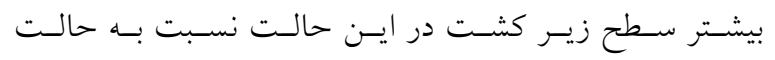

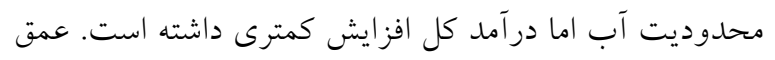

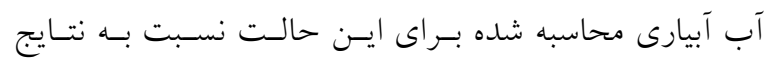

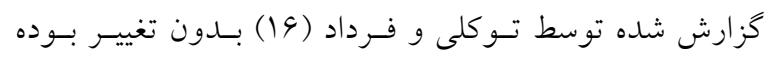

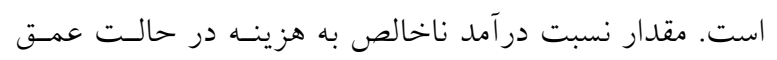

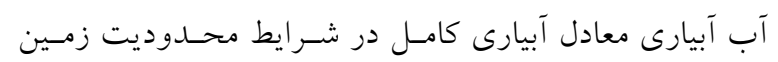

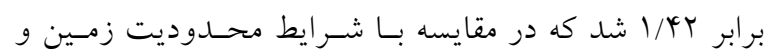
محدوديت آب آبيارى تفاوت جندانى ندارد.

\section{عمق آب آبيارى حالت سربهسرى (Wh)}

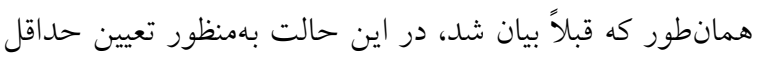

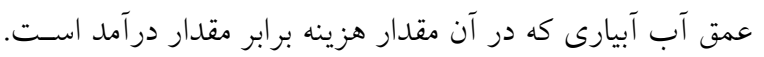

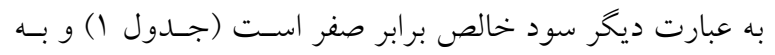

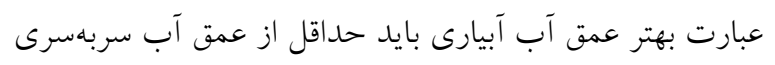

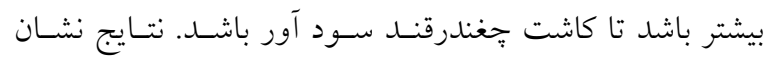

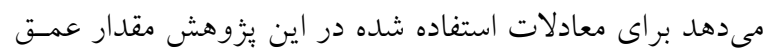

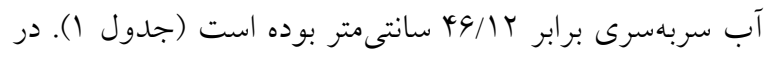

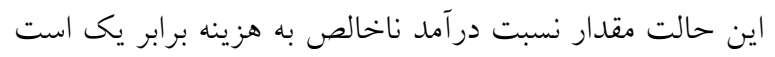

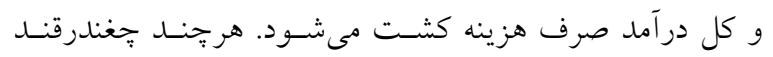

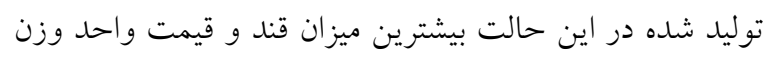

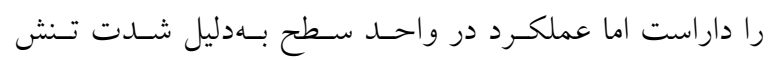
كاهش شديدى دارد.

بررسى اثر دقت ضرايب معادلات محصول، هزينه و قيمت

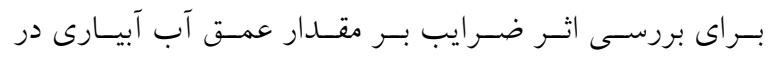

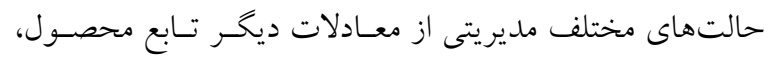

خواهند رفت افزايش مى يابد. نسبت درآمد ناخالص به هزينه در

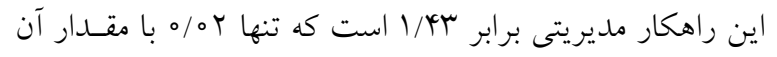

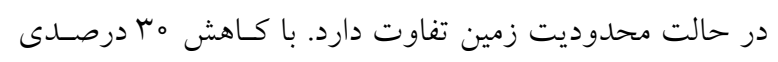

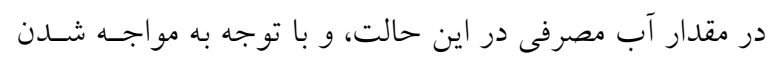

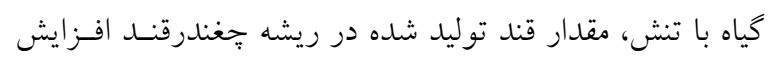

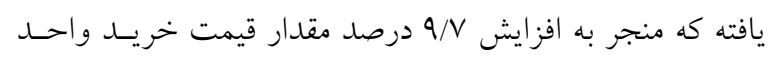

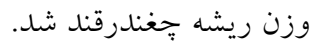

عمق آب آبيارى معادل آبيارى كامل در شـرايط محسدوديت

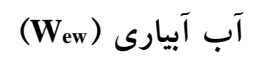
در اين حالت هدف تعيين عمق آب آبيارى است كه در آن سود خالص بهازاى واحد آب مصرفى برابر سـود خـالص در واحســ

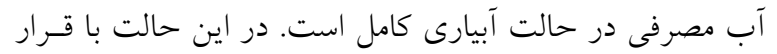

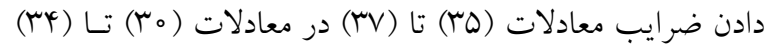

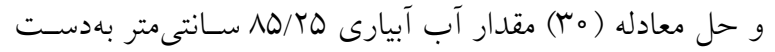

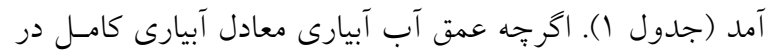

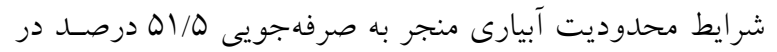

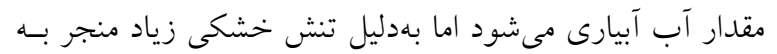

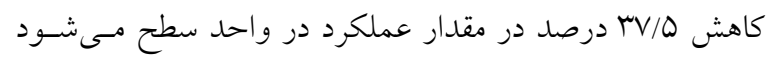

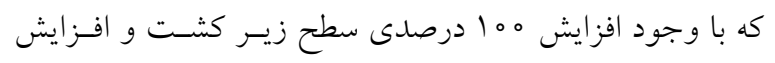
19 درصدى قيمت واحد وزن محصول ريشه جغنندرقند، مقـدار

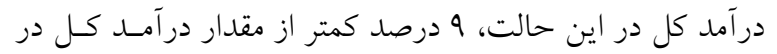

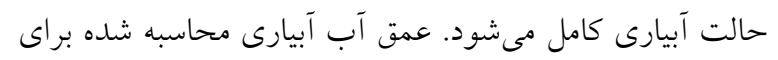

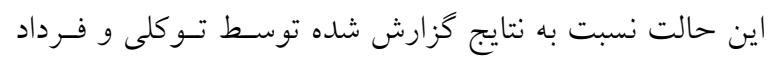
(19) بدون تغيير بوده است. عمق آب آبيارى معادل آبيارى كامل در شـرايط محسدوديت زمين (Wel براى يُاسخ به اين سوال كه بهازاى جهه مقدار عمق آب آبيارى

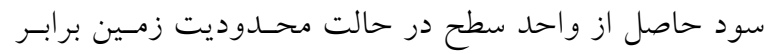

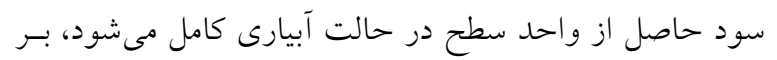

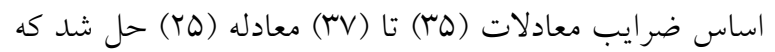




$$
\begin{aligned}
& \text { تأثير دارد. بنابراين تحليلهـاى بـهـار رفتسه در ايسن يـزوهش و } \\
& \text { موارد مشابه ديخر براى هر شرايطى مىبايست ضرايب معادلات }
\end{aligned}
$$

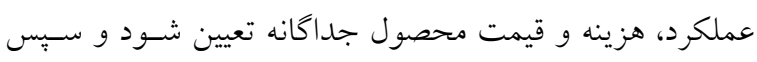

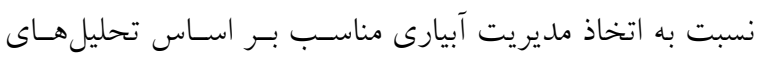

$$
\begin{aligned}
& \text { ارائه شده در اين بيزوهش اقدام كرد. } \\
& \text { نتيجه كيرى }
\end{aligned}
$$

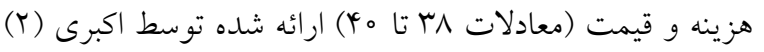

$$
\begin{aligned}
& \text { استفاده شد. نتـايج محاسـبه عمـق آب آبيـارى در راهكارهـاى } \\
& \text { مختلف آبيارى بر اساس توابع توليد، هزينه و قيمت حاصـل از } \\
& \text { يزوهش اكبرى (Y) در جدول (1) آمده است. راهكـار منتخـب } \\
& \text { براى رسيدن به سود حداكثر در شرايط محدوديت زمسين و آب } \\
& \text { مانند نتايج حاصل از كاربرد توابع ارائسه شـده توسـط تـوكلى و } \\
& \text { فرداد (1) است. در اين مورد هم عمق آب آبيـارى در حالـت } \\
& \text { محدوديت زمين منجر به حداكثر سود در واحد سطح مىشود و } \\
& \text { حالت محدوديت آب با احتساب افزايش سطح زير كشت منجر } \\
& \text { به حداكثر درآمد كل مىشود. اما نكتهاى كه بسـيار مهـم اسـت }
\end{aligned}
$$

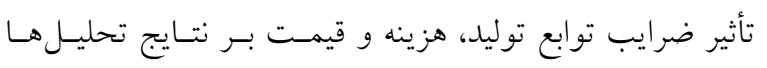

$$
\begin{aligned}
& \text { است. به كونهاى كه در صورت كاربرد معادلات ارائه شده توسط }
\end{aligned}
$$

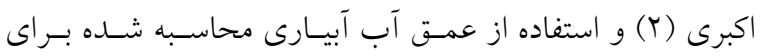

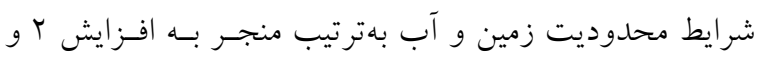

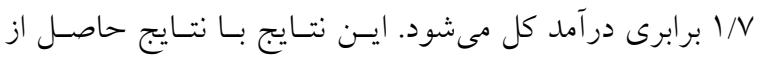

$$
\begin{aligned}
& \text { كاربرد معادلات ارائه شده توسط تـوكلى و فـرداد (4) تفـاوت } \\
& \text { زيادى دارد. تفـاوت ضـرايب توابـع عملكـــد، هزينـه و قيمـت } \\
& \text { محصول بستخى به عوامل مهمى مانند رقـم جغنندرقنـد، عوامـل } \\
& \text { اقليمى، نوع سيستم آبيارى، عوامل زراعى و هـر عـاملى كـهـ بـر } \\
& \text { مقدار محصول بر واحد سطح و هزينه و درصسد قنـد محصـول }
\end{aligned}
$$

منابع مورد استفاده

1. Ahmadi, S. H., M. Agharezaee, A. A. Kamgar-Haghighi and A. R. Sepaskhah. 2014. Effects of dynamic and static deficit and partial root zone drying irrigation strategies on yield tuber sizes distribution, and water productivity of two field grown potato cultivars. Agricultural Water Management 134: 126-136.

2. Akbari, M. Impact of deficit iriigation on sugar beet yield, In: Proceeding of 1999 9th Iranian National Seminar on Irrigation and Drainage, Esfahan, Iran. (in Farsi).

3. English, M. 1990. Deficit irrigation. I: Analytical framework. ASCE. Journal of Irrigation and Drainage Division 116(3): 399-402.

4. English, M. and S. N. Raja. 1996. Perspective on deficit irrigation. Agricultural Water Management 32(1): 1-14.

5. English, M. J., J. T. Musick and V. V. N. Murty. 1990. Deficit irrigation. In: G. J. Hoffman, T. A. Howell and K. H. Solomon (Eds). Management of farm irrigation systems, ASAE Monograph no. 9. American Society of Agricultural Engineers, $1020 \mathrm{p}$.

6. Geerts, S. and D. Raes. 2009. Deficit irrigation as an on- farm strategy to maximize crop water productivity in dry areas. Agricultural Water Management 96: 1275-1284.

7. Hoffmann, C. M. 2010. Root quality of sugar beet. Sugar Technology 12(3-4): 276-287.

8. Jovzi, M. and H. Zare Abyaneh. 2016. Water productivity and water use efficiency indexes of sugar beet under different levels of water and nitrogen fertilizer. Journal of Water and Soil Conservation 22(5): 117-133 (in Farsi).

9. Loel, J., C. Kenter, B. Märländer and C. M. Hoffmann. 2014. Assessment of breeding progress in sugar beet by 
testing old and new varieties under greenhouse and field conditions. European Journal of Agronomy 52: 146-156.

10. Mannocchi, F. and P. Mecarelli. 1994. Optimization analysis of deficit irrigation system. Journal of Irrigation and Drainage Division, ASCE 120(3): 484-503.

11. Pereira, L. S., T. Oweis and A. Zairi. 2002. Irrigation management under water scarcity. Agricultural Water Management 57: 175-206.

12. Sepaskhah, A. R. and D. Akbari. 2005. Deficit irrigation planning under variable seasonal rainfall. Biosystem Engineering 92: 97-106.

13. Sepaskhah, A. R., A. Azizian and A. R. Tavakoli. 2006. Optimal applied water and nitrogen for winter wheat under variable seasonal rainfall and planning scenarios for consequent crops in a semi-arid region. Agricutural Water Management 84(1-2): 113-122.

14. Sepaskhah, A. R., A. R. Tavakoli and S. F. Moosavi. 2006. Principles and Application of Deficit Irrigation. IRICID Publication. (in Farsi).

15. Tarkalson, D. D. and B. A. King. 2017. Effect of deficit irrigation timing on sugarbeet. Agronomy Journal 109(5): 2119-2127.

16. Tavakoli, A. R. and H. Fardad. 1999. Economic evaluation of defecit irrigation on sugarbeet for optimization of water use. Iranian Journal of Agriculture Science 30(3): 575-584. (in Farsi).

17. Topak, R., B. Acar, R. Uyanöz and E. Ceyhan. 2016. Performance of partial root-zone drip irrigation for sugar beet production in a semi-arid area. Agricultural Water Management 176: 180-190.

18. Wu, G. Q., C. M. Wang, Y. Y. Su, J. J. Zhang, R. J. Feng and Lang N. 2014. Assessment of drought tolerance in seedlings of sugar beet (Beta vulgaris L.) cultivars using inorganic and organic solutes accumulation criteria. Soil Science and Plant Nutrition 60: 565-576.

19. Wu, G. Q., R. J. Feng and Q. Z. Shui. 2016. Effect of osmotic stress on growth and osmolytes accumulation in sugar beet (Beta vulgaris L.) plants. Plant Soil Environment 62: 189-194.

20. Wu, G. Q., R. J. Feng, N. Liang, H. J. Yuang and W. B. Sun. 2015: Sodium chloride stimulates growth and alleviates sorbitol-induced osmotic stress in sugar beet seedlings. Plant Growth Regulation 75: 307-316.

21. Zand-Parsa, S. H. and A. R. Sepaskhah. 2001. Optimal applied water and nitrogen for corn. Agricultural Water Management 52: 73-85. 


\title{
Determining the Optimum Applied Water for Sugar Beet in the Case of Crop Price as a Function of Applied Irrigation Water
}

\author{
A. Shabani ${ }^{*}$
}

(Received: June 10-2017 ; Accepted: September 4-2018)

\begin{abstract}
Shortage of irrigation water is a major problem constraining in agricultural production in arid and semi-arid regions. Deficit irrigation is one way to cope with water scarcity and increase water use efficiency. Determining the optimum applied water based on economic analysis is a major key to the deficit irrigation strategy. In this study, the required equations were derived to determine the optimum applied water for sugar beet when crop price is a function of the applied water. The results showed that the optimum applied water under land limiting conditions $(144.98 \mathrm{~cm})$ resulted in the maximum net benefit per unit area (2089741 Rials ha-1). Applying the optimum water depth under land limiting resulted in $17.48 \%$ decrease in the applied water and $15.05 \%$ increase in the total net benefit, in comparison with the maximum yield condition. In water limiting conditions (land is not limiting), the total net benefit was maximized by applying the saved water to put larger areas of land under irrigation. Applying the optimum water depth under water limit condition resulted in 31.2\% decrease in applied water and 45 and 52.36\% increase in the planting area and the total net benefit, in comparison with the maximum yield condition, respectively. Sugar beet planting can be, therefore, profitable if the applied water depth is greater than $67.53 \mathrm{~cm}$ in this study area.
\end{abstract}

Keywords: Economic analysis, Deficit irrigation, Land limiting, Water limit

1. Department of Water Science and Engineering, Faculty of Agriculture, Fasa University, Fasa, Iran.

*: Corresponding Author, Email: shabani8ali@gmail.com 